\title{
Anti-Aquaporin-4 Antibody-Positive Neuromyelitis Optica Presenting with Syndrome of Inappropriate Antidiuretic Hormone Secretion as an Initial Manifestation
}

\author{
H. Nakajima ${ }^{a, b} \quad$ Y. Fujiki $^{a} \quad$ T. Ito $^{a} \quad$ H. Kitaoka ${ }^{a} \quad$ T. Takahashic \\ aDepartment of Internal Medicine, Seikeikai Hospital, Sakai, bepartment of Internal \\ Medicine I, Osaka Medical College, Takatsuki, and 'Department of Neurology, Tohoku \\ University Graduate School of Medicine, Sendai, Japan
}

\section{Key Words}

Neuromyelitis optica - Anti-aquaporin-4 antibody - Syndrome of inappropriate antidiuretic hormone secretion $\cdot$ Hypothalamus

\begin{abstract}
The distribution of neuromyelitis optica (NMO)-characteristic brain lesions corresponds to sites of high aquaporin-4 (AQP4) expression, and the brainstem and hypothalamus lesions that express high levels of AQP4 protein are relatively characteristic of NMO. The syndrome of inappropriate antidiuretic hormone secretion (SIADH) is one of the important causes of hyponatremia and results from an abnormal production or sustained secretion of antidiuretic hormone (ADH). SIADH has been associated with many clinical states or syndromes, and the hypothalamic-neurohypophyseal system regulates the feedback control system for ADH secretion. We report the case of a 63-year-old man with NMO, whose initial manifestation was hyponatremia caused by SIADH. Retrospective analysis revealed that the serum anti-AQP4 antibody was positive, and an MRI scan showed a unilateral lesion in the hypothalamus. SIADH recovered completely with regression of the hypothalamic lesion. As such, NMO should even be considered in patients who develop SIADH and have no optic nerve or spinal cord lesions but have MRI-documented hypothalamic lesions.
\end{abstract}

\section{Introduction}

Neuromyelitis optica (NMO; Devic's disease) is an idiopathic inflammatory disease of the central nervous system that mainly affects the optic nerve and spinal cord. 
Conventionally, NMO is believed to differ from multiple sclerosis (MS) by causing very severe, often bilateral, optic neuritis and longitudinally extensive spinal cord lesions but no brain lesions or aggressive progression to disability and death [1]. Anti-aquaporin-4 (AQP4) antibody was identified as a disease-specific autoantibody in NMO patients [2]. Recent studies have reported a high frequency of magnetic resonance imaging (MRI)documented brain abnormalities in NMO patients. NMO brain lesions characteristically occurred in the hypothalamus, brainstem, or periventricle lesions, which correspond to brain regions with high levels of AQP4 expression $[3,4]$.

The syndrome of inappropriate antidiuretic hormone secretion (SIADH) is one of the important causes of hyponatremia and results from an abnormal production or sustained secretion of antidiuretic hormone (ADH). SIADH has been associated with many clinical states or syndromes, and the hypothalamic-neurohypophyseal system regulates the feedback control system for ADH secretion. Herein, we report a case of a patient with NMO presenting with hyponatremia as an initial manifestation, in whom an MRI scan showed a unilateral lesion in the hypothalamus.

\section{Case Report}

A 63-year-old man had a poor appetite and was found to have severe hyponatremia of $114 \mathrm{mEq} / \mathrm{l}$. He had neither edema nor dehydration. Plasma osmolality was $260 \mathrm{Osm} / \mathrm{kg}$, whereas urinary osmolality was elevated to $436 \mathrm{Osm} / \mathrm{kg}$. Adrenal, renal, and thyroid functions were normal. In addition, he showed no evidence of edema, dehydration, heart failure, or liver cirrhosis. Results of paraneoplastic tests were all normal. These findings indicated SIADH, according to the criteria of Schwartz et al. [5]. A brain MRI showed no abnormality in the pituitary, although it showed a nonenhanced T2-weighted lesion in the hypothalamus ( $\underline{\text { fig. 1 }}$ a, b). T1- and diffusion-weighted brain images showed no abnormalities. His serum sodium levels were restored by water restriction. During this episode, no corticosteroid or other immunosuppressive therapy was offered. Two months later, his serum sodium levels completely recovered without water restriction. Three months after the initial episode of SIADH, the patient developed numbness in his legs and spinal ataxia. Neurological examination showed a positive Romberg's sign and sensory disturbances below the C5 level on the right side and below the T10 level on the left side. His muscle strength was 1/5 in the legs, and Babinski signs were negative. MRI of the brain showed no additional lesion, and the hypothalamic lesion was markedly diminished 3 months after the SIADH episode (fig. 1c). Spinal MRI revealed long lesions extending from the upper cervical spinal cord to the thoracic cord (fig. 1d). A cerebrospinal fluid study showed pleocytosis $\left(24 / \mathrm{mm}^{3}\right)$, a normal protein level (43 mg/dl), and no oligoclonal IgG bands. The serum was positive for anti-AQP4 antibody $(1: 1,024)$, as revealed by a sensitive detection method [6]. He also had an elevated titer of antinuclear antibodies (1:80), anti-SS-A antibody $(185 \mathrm{U} / \mathrm{ml})$, and the cytoplasmic antineutrophil cytoplasmic antibody (C-ANCA; $78 \mathrm{EU}$ ). However, he did not show any clinical features of Sjögren syndrome (SS) or Wegener granulomatosis, and the other serum antibodies such as anti-SS-B, anti-dsDNA, anti-Sm, and anti-RNP antibodies, and the perinuclear antineutrophil cytoplasmic antibody (P-ANCA) were negative. Thus, the patient was diagnosed as having NMO spectrum disorder. He was successfully treated with high-dose methylprednisolone (a 6-day course of 1,000 mg methylprednisolone) and immunoadsorption plasmapheresis, followed by once-daily prednisolone $(20 \mathrm{mg})$. Follow-up serum examination showed a $99.2 \%$ decrease in the anti-AQP4 antibody titer (1:8). The anti-SS-A antibody titer also decreased $(44.5 \mathrm{U} / \mathrm{ml})$, and the C-ANCA changed to negative. He has not had any additional relapses for 2 years after disease onset by taking $10 \mathrm{mg}$ of prednisolone daily.

\section{Discussion}

SIADH is a condition caused by an excessive secretion of $\mathrm{ADH}$ or $\mathrm{ADH}$-like substances. Dysfunction of the hypothalamic-pituitary axis, which plays a central role in 
the regulation of $\mathrm{ADH}$ secretion, is responsible for SIADH. SIADH was originally described by Schwartz et al. [5] in 2 patients with bronchogenic carcinoma who had severe hyponatremia at presentation. The most common causes of SIADH are malignancy, pulmonary disorders, central nervous system disease, and medication. As to intracranial diseases, SIADH may also be manifested in cases of traumatic brain injury, brain tumors, subarachnoid hemorrhage, or infectious disease. Several studies have reported cases of MS patients presenting with SIADH [7-11]. Tsui et al. [11] have described a case of MS with SIADH in a 48-year-old female who presented with bilateral hypothalamic lesions on brain MRI. Sakai et al. [9] have reported an autopsied case of SIADH associated with MS that showed a loss of neuronal cells of the supraoptic nuclei in the hypothalamus. In this case, the demyelinating lesions may have secondarily affected the supraoptic and paraventricular nucleus of the hypothalamus, causing ADH leakage, which could then have triggered the SIADH. As to NMO, Lotze et al. [12] reported on a 15-year-old girl with AQP4 antibody-positive NMO who had symptomatic hyponatremia caused by SIADH attributed to disease involvement of the bilateral hypothalamus on brain MRI. She was treated initially with intravenous methylprednisolone for 5 days, followed by a 6-week oral steroid taper with resolution of her paresthesias caused by cervical myelitis and SIADH. You et al. [13] reported on a 56-year-old woman with AQP4 antibody-positive NMO who had symptomatic hyponatremia caused by SIADH. Her brain MRI showed no hypothalamic lesion, but it was speculated that there may have been damage to the tiny connective fibers of the hypothalamus that could not be detected by MRI [13]. The present case was characteristic in that it started with SIADH, and in addition, a unilateral hypothalamic lesion was detected by brain MRI. SIADH recovered completely with regression of the hypothalamic lesion. Although abundant in the optic nerve and spinal cord, AQP4 is found throughout the brain. In addition, AQP4 expression has been shown to be higher in the hypothalamic and periventricular areas of the brain [3]. Thus, SIADH may have been caused by the anti-AQP4 antibody-mediated inflammation in the hypothalamus. The hypothalamus controls body temperature, hunger, thirst, fatigue, sleep, and circadian cycles by the secretion of oxytocin, vasopressin, or other hypothalamic-releasing hormones. Baba et al. [14] have reported on a patient with NMO spectrum disorder that was complicated by narcolepsy associated with bilateral hypothalamic lesions and a low hypocretin cerebrospinal fluid level. Thus, NMO spectrum disorder accompanied by hypothalamic lesions may be indicative of a disorder of an endogenous circadian rhythm as a manifestation of hypothalamic lesions.

NMO patients often have an accompanying autoimmune disease, such as SS, systemic lupus erythematosus, or a related profile of non-organ-specific autoantibodies. However, the anti-AQP4 antibodies would be directly related to the neurologic process [15]. Recent case reports described patients with SS or systemic lupus erythematosus presenting with NMO spectrum disorder as initial presentation $[16,17]$. In these cases, checking for the presence of anti-AQP4 antibodies provides important prognostic information and may help to guide treatment decisions.

In conclusion, we reported the case of a patient with NMO spectrum disorder who showed SIADH associated with a unilateral hypothalamic lesion as an initial manifestation. NMO should be considered even in patients who develop SIADH and have no optic nerve or spinal cord lesions but have MRI-documented hypothalamic lesions. 


\section{Acknowledgement}

We are grateful to the patient for the permission to publish this information.

\section{Disclosure Statement}

The authors declare that they have no competing interests. There are no financial conflicts of interest in this study.
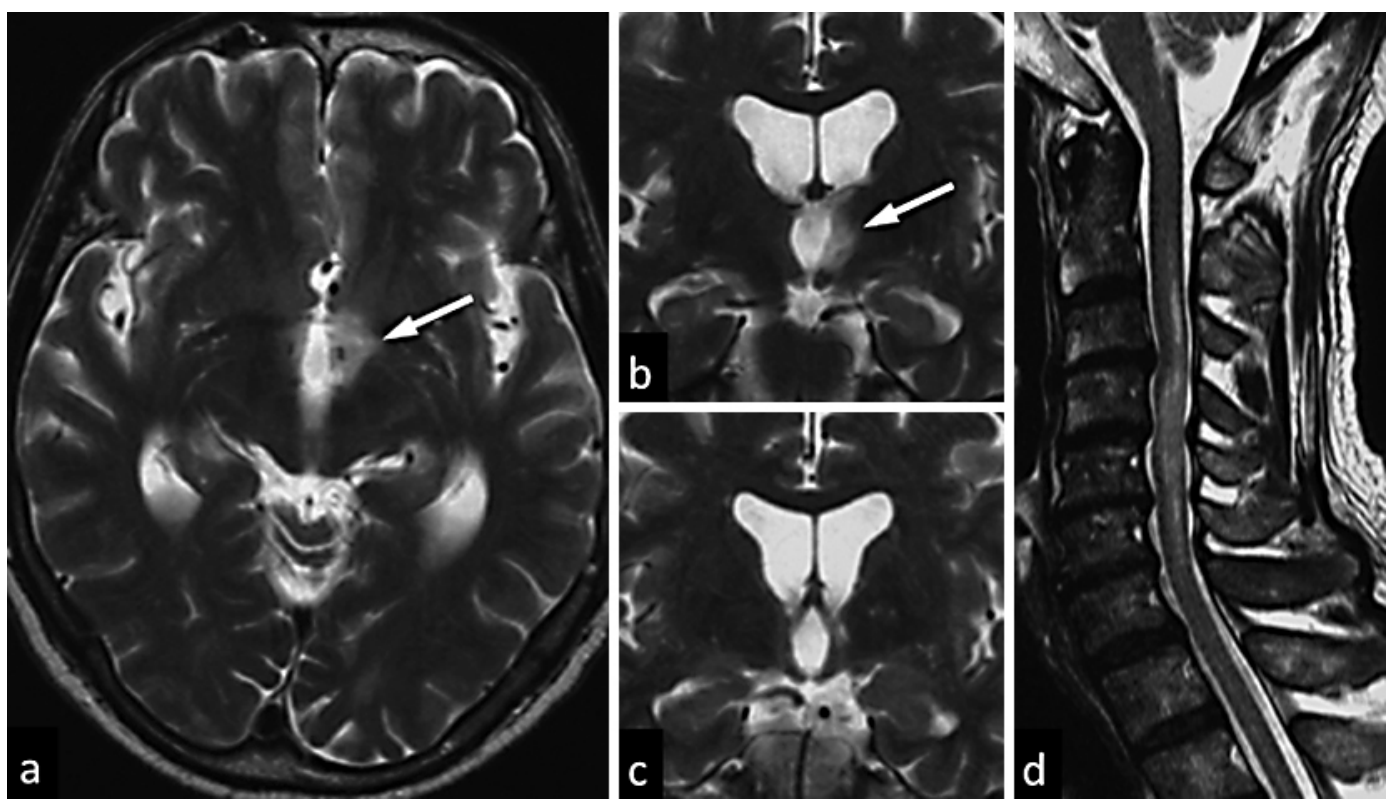

Fig. 1. MRI findings of the present case. A hypothalamic lesion (arrow) was observed during the SIADH episode. Axial and coronal sections of a T2-weighted image showed marked hyperintense lesions at the hypothalamus $(\mathbf{a}, \mathbf{b})$. The hypothalamic lesion was markedly diminished during a relapse of acute myelitis 3 months after the SIADH episode (c). A sagittal T2-weighted image showed a long spinal cord lesion with high signal intensity during a relapse of acute myelitis $(\mathbf{d})$.

\section{References}

1 Wingerchuk DM, Lennon VA, Lucchinetti CF, Pittock SJ, Weinshenker BG: The spectrum of neuromyelitis optica. Lancet Neurol 2007;6:805-815.

2 Lennon VA, Kryzer TJ, Pittock SJ, Verkman AS, Hinson SR: IgG marker of optic-spinal multiple sclerosis binds to the aquaporin-4 water channel. J Exp Med 2005;202:473-477.

3 Pittock SJ, Weinshenker BG, Lucchinetti CF, Wingerchuk DM, Corboy JR, Lennon VA: Neuromyelitis optica brain lesions localized at sites of high aquaporin 4 expression. Arch Neurol 2006;63:964-968.

4 Nakashima I, Fujihara K, Miyazawa I, Misu T, Narikawa K, Nakamura M, Watanabe S, Takahashi T, Nishiyama S, Shiga Y, Sato S, Weinshenker BG, Itoyama Y: Clinical and MRI features of Japanese patients with multiple sclerosis positive for NMO-IgG. J Neurol Neurosurg Psychiatry 2006;77:1073-1075. 
5 Schwartz WB, Bennett W, Curelop S, Bartter FC: A syndrome of renal sodium loss and hyponatremia probably resulting from inappropriate secretion of antidiuretic hormone. American Journal of Medicine 1957;23:529542.

-6 Takahashi T, Fujihara K, Nakashima I, Misu T, Miyazawa I, Nakamura M, Watanabe S, Shiga Y, Kanaoka C, Fujimori J, Sato S, Itoyama Y: Anti-aquaporin-4 antibody is involved in the pathogenesis of NMO: a study on antibody titre. Brain 2007;130:1235-1243.

7 Apple D, Kreines K, Biehl JP: The syndrome of inappropriate antidiuretic hormone secretion in multiple sclerosis. Arch Intern Med 1978;138:1713-1714.

-8 Ishikawa E, Ohgo S, Nakatsuru K, Yamamura Y, Nagamine M, Kuribayashi T, Matsukura S: Syndrome of inappropriate secretion of antidiuretic hormone (SIADH) in a patient with multiple sclerosis. Jpn J Med 1989;28:75-79.

-9 Sakai N, Miyajima H, Shimizu T, Arai K: Syndrome of inappropriate secretion of antidiuretic hormone associated with multiple sclerosis. Intern Med 1992;31:463-466.

10 Liamis G, Elisaf M: Syndrome of inappropriate antidiuresis associated with multiple sclerosis. J Neurol Sci 2000;172:38-40.

11 Tsui EY, Yip SF, Ng SH, Cheung YK: Reversible MRI changes of hypothalamus in a multiple sclerosis patient with homeostatic disturbances. Eur Radiol 2002;12:S28-S31.

12 Lotze TE, Northrop JL, Hutton GJ, Ross B, Schiffman JS, Hunter JV: Spectrum of pediatric neuromyelitis optica. Pediatrics 2008;122:e1039-e1047.

13 You XF, Qin W, Hu WL: Aquaporin-4 antibody positive neuromyelitis optica with syndrome of inappropriate antidiuretic hormone secretion. Neurosciences (Riyadh) 2011;16:68-71.

14 Baba T, Nakashima I, Kanbayashi T, Konno M, Takahashi T, Fujihara K, Misu T, Takeda A, Shiga Y, Ogawa H, Itoyama Y: Narcolepsy as an initial manifestation of neuromyelitis optica with anti-aquaporin-4 antibody. J Neurol 2009;256:287-288.

-15 Pittock SJ, Lennon VA, de Seze J, Vermersch P, Homburger HA, Wingerchuk DM, Lucchinetti CF, Zéphir H, Moder K, Weinshenker BG: Neuromyelitis optica and non organ-specific autoimmunity. Arch Neurol 2008;65:78-83.

-16 Karim S, Majithia V: Devic's syndrome as initial presentation of systemic lupus erythematosus. Am J Med Sci 2009;338:245-247.

17 Kahlenberg JM: Neuromyelitis optica spectrum disorder as an initial presentation of primary Sjögren's syndrome. Semin Arthritis Rheum 2011;40:343-348. 